\title{
Artefact integration by concurrent enterprises and productive break-up
}

\author{
R.C. Michelini, G.M. Acaccia, M. Callegari, \\ R.M. Molfino and R.P. Razzoli \\ DIMEC: Industrial Robot Design Research Group \\ University of Genova \\ Via all'Opera Pia 15A, 16145 Genova, Italy \\ Tel.+39103532857, Fax +39103532974 \\ e-mail:pmar@dimec.unige.it
}

\begin{abstract}
The investigation is based on the activity performed along the past years by the Industrial Robot Design Research Group at the University of Genova; it discusses results carried on at the shop-floor level and example implementations are shown, comparing typical lay-outs for the automatic assembly of varying products mixes. Basically, the modular assembly lines, whose versatility is fixed at the strategic level by modifying the configuration, are distinguished from the robotised assembly cells, having on-line versatility due to their functional adaptivity. The situations are referred to example cases faced by small to medium size companies. The results are acknowledged exploring the plant effectiveness by means of expert simulation, used, during the development stages, for setting the facilities lay-out and, during the running stages, for fitting the facility govern.
\end{abstract}

\section{Keywords}

Integrated manufacturing, economy of scope, productive break up

\section{INTRODUCTION}

The manufacturing enterprises face the need of relevant reorganisations to preserve competitiveness in a world-wide context, where information technologies supply the support for function integration by means of knowledge intensive work- 
organisation set-ups. Changes aim at 'integrated' approaches, to obtain artefacts which incorporate purpose-driven components, to make possible the transfer of the mix of properties, exactly matching the oriented requests of any particular buyer. The aim of customised products within industrial businesses establishes according to the 'economy of scope' rules and is the issue of relevant innovations such as: - concentration of investment strictly into resources needed by the work-cycles; - effectiveness improvement by technological versatility and adaptive schedules;

- resort to facilities flexibility to remove function and equipment redundancy; - integrated management-and-control support for just-in-time manufacturing; - break up of the flow-shop, with decentralised production into autonomous units; - empowerment of involved workers, with decision proxy where problems arise; - benchmarking of reached results, with recognition of individual successfulness; - etc.

The success of the 'new' organisation depends on 'leanness', focusing the business resources only on the basic manufacturing process concerned by the transformation of materials and components into final artefacts with augmented saleable value and, at the same time, suppressing side accomplishments which do not increase the items 'objective' value. The paper moves from this kind of topics and looks, more specifically, to the integration steps for part composition. Production agendas are the outcome of special manufacturing segments, to be unified by knowledge sharing. The solution leads to the 'intelligent' factory, based on distributed information lay-out, supporting the integrated control and management of the production facilities.

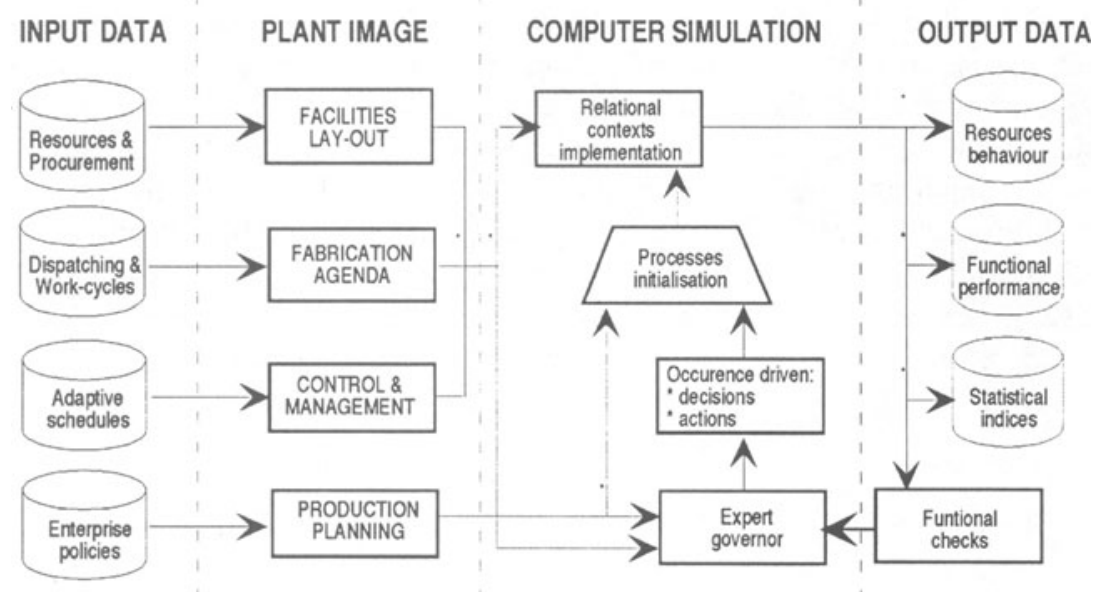

Figure 1. Reference setting of an expert simulator 


\section{ANALYSING EFFECTIVENESS BY VIRTUAL TESTING}

The integrated control and management set-ups, aiming at return on investment by flexible specialisation and simultaneous engineering through the economy of 'scope', do not correspond to clear-cut specifications and their effectiveness is actually proved only after the experimentation with real running conditions. The direct reference to existing facilities and practically implemented production programmes is a valuable help, as well as the exploitation of prototypal pilot plantsn since, each time, particular aspects can be appreciated, with evidence of the contingencies. This cannot avoid risky business, as soon as the innovating pace tries not yet explored tracks. The suitable functional modelling of the innovation and the virtual testing by expert simulation, Figure 1, are the basic support of production engineers; the resulting codes exploit: - causal frames, typically to deal with the physical resources transformation, by means of algorithmic blocks; and: judgmental frames, to emulate the decisional logics governing the processes, by means of heuristic blocks.

During the development stage of a new manufacturing facility, the virtual testing allows comparative evaluations between competing lay-outs and between alternative steering policies; then, simulation is made to run in compressed time and statistical restitution is enabled to work out series of performance indices. Then, the intelligent manufacturing characterises by the life-long resort to simulation aids, to set (strategic, tactical and executional) flexibility, adapting the schedules to improve plant efficiency. The "value chain" model is established, once the achieved performance is measured: every investment is assessed on its ability of creating benefits in terms of customers' satisfaction value, added to the artefact, during processing. The conditioning contexts are by now prettily well acknowledged, with implementation lines properly identified and hints for maximising the return on investment in terms of business organisation fitting out.

Example opportunities are mentioned with the present discussion, centring the attention on the manufacturing accomplishments pertaining to assembling tasks. By overseeing the artefact integration, an enterprise can reach customer's satisfaction by incorporating specialised mastership, according to the rules of the productive break-up, with allocation of the development and processing tasks to the owners of the qualifying technologies for the efficient manufacturing of each part.

\section{COMBINING SUB-ASSEMBLIES INTO COMPLEX ARTEFACTS}

On these premises, 'intelligent' enterprises develop, aiming at products or services customisation to widen offers for individual needs or whims satisfaction, by means of integrating components and technologies according to sectorialised goals. The artefact, thereafter, is progressively becoming a 'special' build-up (grounded, e.g., 
on 'mechatronics' concepts), obtained by composing subsets of items, each one developed by specialists of a given field. The winning enterprise is, finally, an integrator, responsible of the "fitness-with-purpose" achievement of final items, even if choice and development of the components remain critical steps, since:

- the selection of the sub-assemblies technology is main contrivance to efficiently obtain that offer diversification will reach the optimal quality/price ratio (by respect to world-wide competing enterprises) and will grant 'exactly' the (expressed or not) consumer's demands;

- the design of each part should progress, keeping the integration product-process as central reason, to transparently provide the documentation of the delivered quality (for its technical certification) and to enable the economy of scope by cooperative knowledge processing.

The comments that follow are related to the composition steps of simple artefacts, with attention on sample cases commonly faced by the industrial practice. The usual technologies are shortly reviewed, then typical situations are presented as explanatory applications and positive conclusions.

\subsection{The technologies of mechanical assembling}

The manufacturing phase of assembly considers a reference 'piece' (with or without supporting pallet) and series of 'parts' to be connected (with or without aid of auxiliary rigs); the task location can move (assembly chain) or can be fixed (assembly stand). The integrated automation can be devised starting from:

- the 'programmable' assembly line, with the addition of information systems for process-and-product monitoring and with real time management of quality data, according to proper adaptive flexibility rules;

- the 'robotised' assembly cell, with the addition of special purpose fixtures to enhance productivity (along with versatility), in order to improve effectiveness as soon as the time-varying product mix is scheduled.

The first set-up privileges the flow-shop organisation and assures high productivity, with shortest crossing time, whether steady artefact specification is maintained. Transfer rigs and special purpose processing units are optimal choices. The pursuit of flexibility reduces productivity, since technological versatility and goal-oriented optimisation contrasts each-other; the strict work-cycle seriality, moreover, can be critical in front of discontinuities (unexpected occurrences or scheduled singularities) or when the variability of the assembly cycles makes ineffective the limitation to prearranged material flows. 


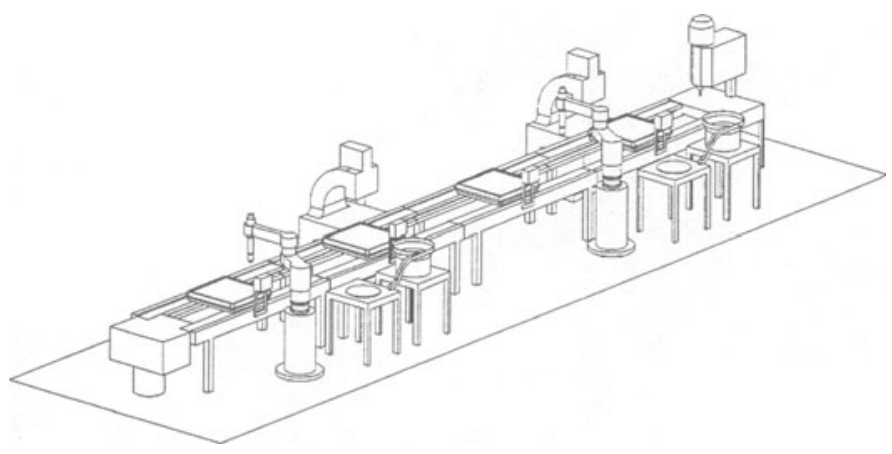

Figure 2. High productivity modular assembly line

On the other hand, the productivity of the robotised lay-out is greatly affected by the material logistics of the job-shop organisation; the effectiveness, thereafter, suffers technologic thresholds (accuracy vs. versatility, dexterity vs. efficiency, etc. are conflicting attributes) and practical hindrances (fixturing, fitting, programming, etc. require life-long up-dating and time consuming commitments). The material flow management, finally, faces demanding accomplishments, for continuously adapting the shop logistics in order to maximise the duty ratio of resources and to minimise the current work-in-progress.

The two approaches are, therefore, both in use, being, each-other subsidiary. Intermediate lay-outs, in case, are also exploited, such as:

- 'short' assembly lines, with forks, sideways and junctions;

- 'dedicated' assembly sections, with local area handling and dispatching.

The proper choice aims at balancing the technical requirements against the return on investment and, as general rule, to following alternatives distinguish:

- resort to modularity in terms of physical resources, Figure 2: the versatility is managed off-line, since along the tactical horizon, the product mix is constant and special purpose fixtures are consistent with the scheduled fabrication agendas; the switching to new products means resetting the modules into a different lay-out, so that the same units are amortised over several product mixes, while compressing the time-to-market due to the consistency of the common equipment;

- resort to modularity in terms of functional resources, Figure 3: the flexibility is enabled to operate on-line, with integrated control-and-management of process facilities; the schedules adapt in real time to reach the delivery times, avoiding redundancies and looking to just-in-time policies, by means of re-assigning duties, once acknowledged the current priorities and supported the proactive maintenance frame for the (subset of) functions, timely required by the business policy. 


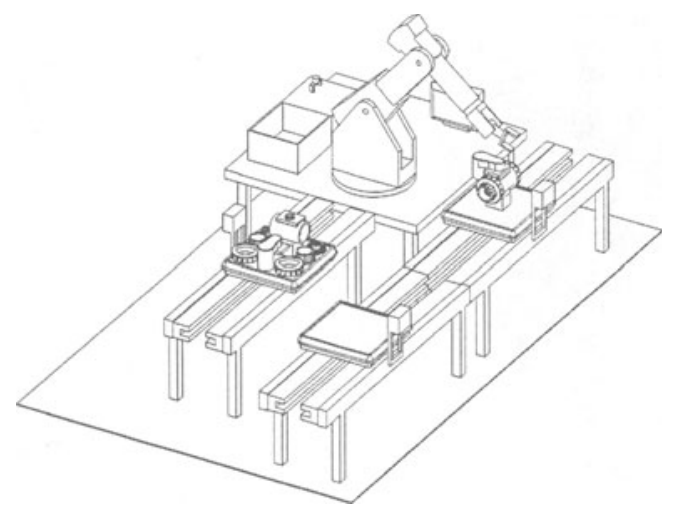

Figure 3. High versatility robot assembly cell

One alternative is effective in front of large batches of low price items, so that the return depends on productivity; the lay-out resetting is performed in parallel with the redesign of new artefacts, minimising the time-to-market with the rules of simultaneous engineering. Figure 4 shows an example outfit, which is properly designed as featuring device to distinguish different product lines, but, at the same time, has to be manufactured by batches of some millions items, to achieve the competitiveness threshold. Modularity of the physical equipment might be pushed at different levels of definition and is certainly dependent to a great extent on the product typology; the fact is largely accepted, but the related options look to be rather unexplored. The other alternative corresponds to high technologic versatility resources and to self-adaptive governing architectures; customer-driven quality and quick response delivery are consistent with one-of-a-kind products and return depends on satisfying quite oriented properties.

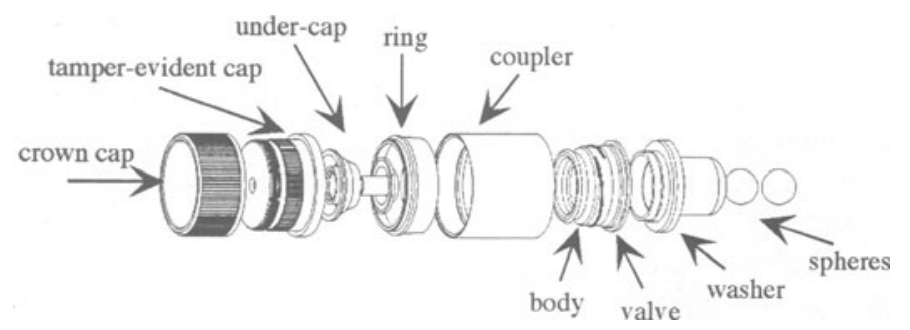

Figure 4. Non-refillable closure for bottles

The supporting decision logic is obtained with resort to knowledge-based architectures, using expert-blocks to implement heuristic data processing and to 
govern the assembling process with plausibility rules assessed by empirical checks; the option, widely used by the traditional craft-work, is being rediscovered by 'intelligent' manufacturing, but actual implementations look to be late in showing real effectiveness, unless for properly structured situations. Figure 5, for instance, presents a rather simple servo-valve that is obtained by combining purpose adapted components (motor, throttle valve, interfaces), depending on clients' request; the schedules are concerned by small batches, making on-line monitoring and unmanned solutions consistent with preserving the expected quality delivery.

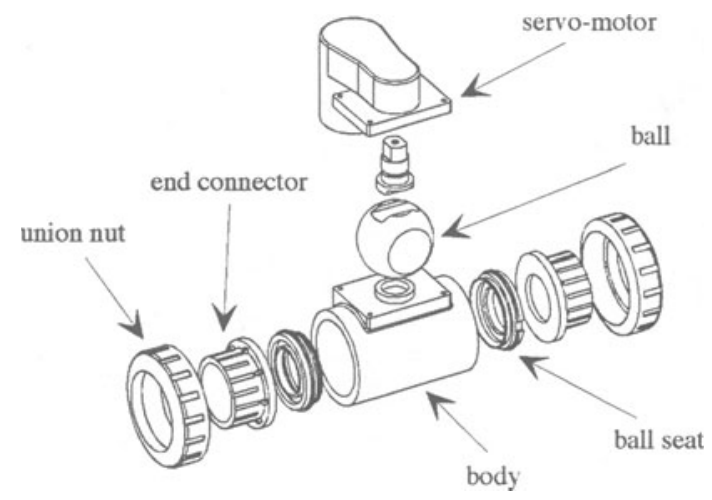

Figure 5. PVC servo-valve for air conditioning plants

The practical selection of the 'best' physical and logic resources and of the 'optimally' balanced settings and schedulings is performed by referring, only, on assessments, confirmed by 'on-the-field' experimentation; optimality is, possibly, even a non-sense, since technology is continuously changing. A worthy approach will, then, aim at looking for effectiveness by fixing a subset of rules to understand how flexibility has to be exploited. The couple of mentioned sample cases is used to introduce explanatory hints. The first development case, as recalled, deals with low price artefacts achieving competitivity through mass-production; still fashions are fast changing and quick responsiveness is needed for winning the market; modularity and lay-out resetting with special purpose units seem to be appropriate option. The second development considers small batch products, timely manufactured as soon as individual users appear, requesting just-in-time delivery, pertaining to repetitive models series; robot assembly cells are effective aid for the automatic release of product data; investment is, only, to be checked against benefits in terms of cost balances and quality warrants. 


\section{EXAMPLE RESULTS AND COMMENTS}

The example studies can be expanded to cover the diversified possibilities of actually feasible situations by using the virtual testing approach based on computer simulation. The extended series of expert simulators SIFIP, described in (Acaccia et al. 1989), was developed by the Industrial Robot Design Research Group of the University of Genova, Italy, exactly to that goal. Referring to the two recalled sample developments, the packages MAA-SIFIP (Acaccia et al., 1996) and XASSIFIP (Michelini et al., 1996) are, respectively, the basic reference environments. The first has been developed using the programming tool MODSIM III by CACI, with proper exploitation of the object-oriented coding, to simplify updating at each facility resetting. The second is based on the expert shell G2 by GENSYM, to grant real-time operativity of the governing block, which has to be transferred to become operative on-line, once the real plant is built.

Let first refer to the assembling of bottle closures, Figure 3; a modular assembling arrangement based on special purpose units, Figure 2, grants the productivity figures consistent with the batch delivering; rearranging the units differently, a new assembly cycle is obtained, each time with optimal schedules on the current tactical horizons. The lay-out is tuned for a given product, removing the on-line flexibility; assembly effectiveness immediately decreases when the line has to deal concurrently with items requiring different assembly cycles. As case arises, the lay-out might be modified, with branching points; by simulation, any change and addition can be investigated, about the effects it rises.

During simulation run-time the animation shows a dynamic representation of main events occurring in the line, with pop-up windows that allow the user to gain a deeper insight in the status of all resources (Figure 6). At the end of simulation, cumulated performance figures are updated and statistical restitution blocks are available to compute effectiveness indices to rank the chosen schedules (Figure 7).

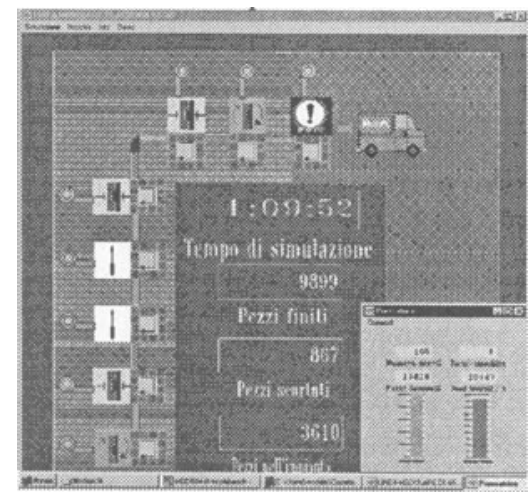

Figure 6. Layout of the production line during animation 
In the setting of the line, several different configurations can be exploited: for instance 7 functional modules can be used, two of which should be very specialised, or 8 functional modules, all of which performing rather standard operations (click-joining or spring locking, ultrasonic soldering, screw linking, tails cutting, glue sticking, fluid leakage testing); some parameters must be tuned to obtain best performance from the line, like speed and capacity of transport units (i.e. velocity and length of conveyor belts), stops management policy of functional units in case of input or output parts shortage or excess; finally, the sensitivity of the system to MTBF and MTTR can be exploited off-line, to select proper components or maintenance policies. Figure 8, for instance, shows line throughput and utilisation ratio for different set-ups of the line (in particular, some conveyors' capacities are varied for the 7 units case).

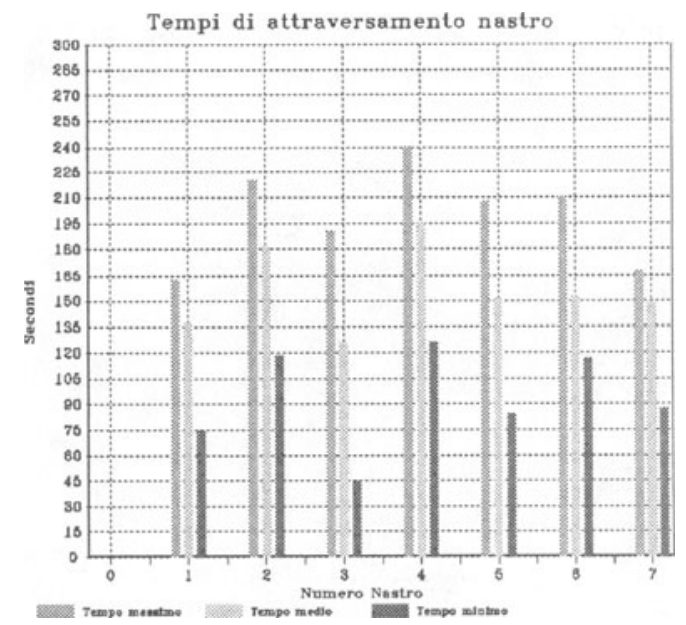

Figure 7. Statistical indices at end of simulation
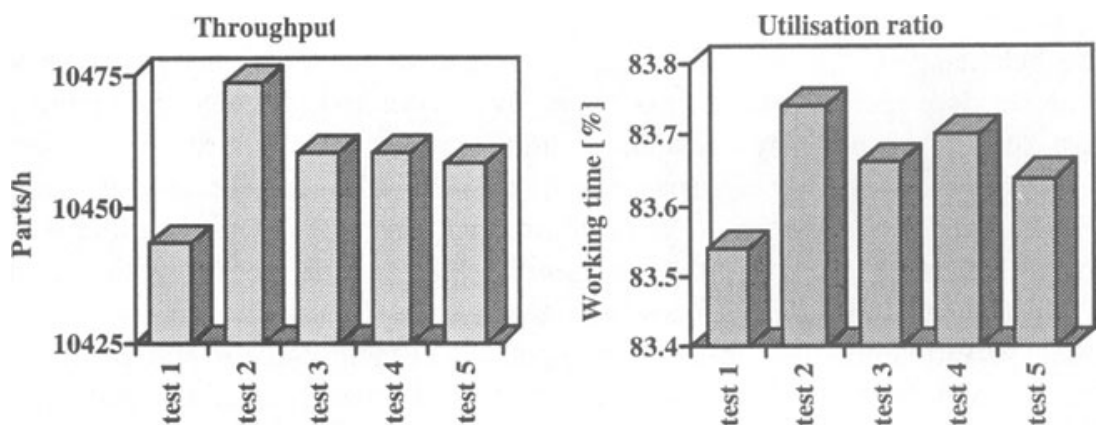

Figure 8. Off-line analysis to compare the performances of different test cases 
The characterisation of flexible assembly based on robot manipulation, Figure 3 , is facing more subtle requirements, as efficiency is reached by adaptive scheduling, enabled via real-time governing logic. The example servo-valves, Figure 4, have to be purposely assembled according to the specification of the case. With a just-intime policy, the manufacturing starts on the condition to have a buyer, but reliable due times are needed, assuring the artefact data, in front of the varying requests. When items variability is very broad, the production strategies, based on feeding the internal warehouse, become ineffective also in terms of storing and sorting requirements. The possibility of exploiting flexibility and quick response manufacturing set-ups has, on the contrary, to be considered as means to manage the changes with no obligation of accepting only pre-set itemisations. In fact, the considered robot stands assure the automatic assembly of the items, in view of any particular end configuration; productivity might be low, still compliance with quality data transparency and with due time has strictly to be observed and assembling has to be pursued during unattended work-shifts whenever required by the individual order.

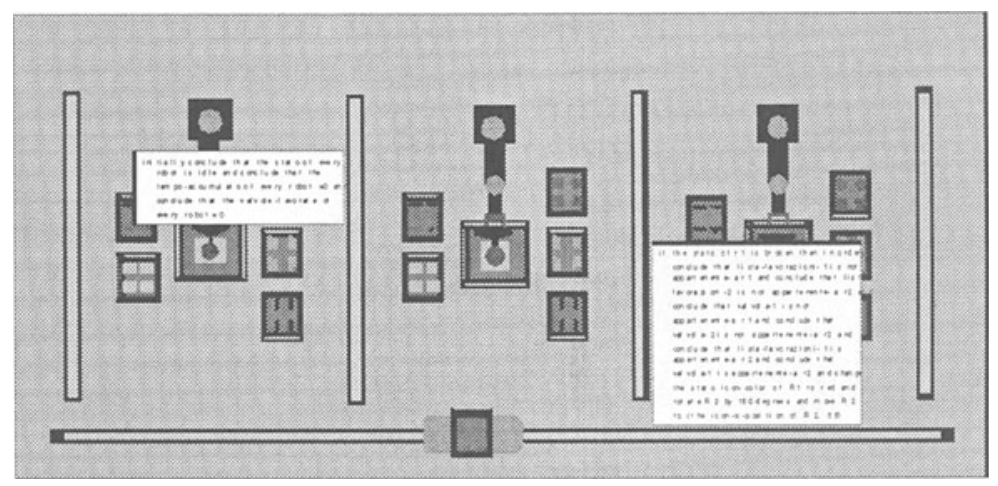

Figure 9. Simulator layout with output workspaces for data display

The balancing of the manufacturing agendas leads to an assembly section with three stands, Figure 9, each one operated by a robot and provided with automatic parts feeders supplied by a common shuttle operated transporter. Each robot is devoted to the mounting of a specific valve series, unless the special setting of the priority indices modify the on-going attribution. The supervisor should check the priority setting and the due date requirements for the delivering of the assorted batch. The parts feeding is pre-set through the shop-floor dispatching service. The robots perform either the picking of components or their insertion and locking.

The complexity of the situations faced for the integrated control-and-management of the example assembly process requires a comparatively sophisticated programming aid. The knowledge-based tool G2 was therefore used, since it possesses very friendly access and it is further capable of supporting real-time 
operations. This means that the expert governor enabled for the simulation, when directly interfaced to the physical facility, performs the control-and-management actions of the selected policy (Figure 10).

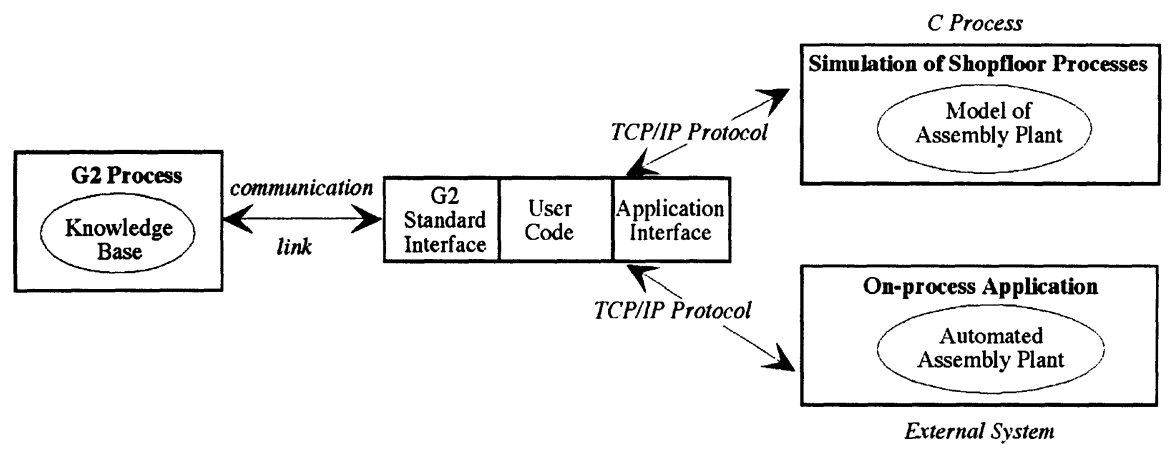

Figure 10. XAS·SIFIP set-up: real-time control and simulation driven scheduler

By means of extensive off-line simulation, attention is focused on:

- robot characteristics: tasks are analysed and overall duty times are acknowledged as averaged figures (details are omitted once teaching is completed);

- failure patterns: the recovery strategies are investigated for different breakdown or damage situations and for changing the workstations priorities;

- compositions of mixes: the (three) artefacts have different assembly times, thus effectiveness depends on the delivery agendas assortments.

The govern fitting is performed exploitating the expert-code XAS-SIFIP; this includes the sub-sets of blocks for on-process and on-line operations, later transferred, as factory automation software, to supervise to the real plant operations. Once a design-feedback-choice cycle is completed, the governor is ready to operate on-line. The basic requirement, distinguishing the on-process exploitation of flexibility from the previously recalled set-up phase, is the need of real time operativity. With the knowledge-based architectures of the expert simulator XAS-SIFIP, developed by means of the shell G2, a twofold advantage arises:

- the implementation of heuristic blocks is straightforward and the emulation of human-like decisional platforms provides the software instruments for enabling the real-time factory automation with govern-for-flexibility features;

- the goal: 'flexible automation' is coherently obtained by merging patterns of action and patterns of structure, provided that structures carry the signature of the originating changes and actions grant the consistency of the generated configurations. 


\section{CONCLUSIONS}

The sketched options are the issue of several factors, conditioning the integration of the customised items with concern of the considered cases and as common spur of the flexible automation. The example situations, first of all, distinguish, when the 'added value' cycles, assuring enterprises' profitability, are compared. The items, mentioned by the first case, while cheap, are result of a proprietary technology, which makes the assembly duty effective; the modular processing units perform simple jobs (click joining, spring locking, screw linking, tails cutting, glue sticking, ultrasonic soldering, etc.) properly enabled once the component parts are redesigned attuning the functions given in charge to the delivered devices and the operations required to accomplish the assembling. The success mainly depends on simultaneous engineering, by balancing product and process design, with emphasis on the modularity of the facility to reach quick response, in view of 'new' artefacts, readily delivered with the effectiveness of mass-production.

When, alternatively, time-varying mixes of artefacts shall be processed, the practice has the habit to look for productive break-up, in front of the frustrating situation of moving 'outside' the manufacturing phases, that, carried 'inside', would downgrade the main business competitiveness. The servo-valves, mentioned as second case, are obtained by joining items supplied by different manufacturers, to yield application-driven devices, suited as special purpose implements. Value cycle monitoring follows, this time, a track highly dependent on the integration of the involved enterprises functions and on the ability of sharing their quality data set-ups; in fact, the approach is becoming quite common, giving rise to efficient 'integrators', namely, 'concept' businesses based on 'external' manufacturing duties; the cooperating network needs be turned to creation of 'added' value and the resulting setting is highly subject to the entrepreneuring attitude, with the possibility of fast re-aggregation of a network around a new leader. The considerations open the discussion on the generalities about integration in intelligent manufacturing.

The artefacts obtained by the selective incorporation of the appropriate technologies move across production programmes obtained with the connection of development and processing segments pertaining to rather diversified business realities, which, nether the less, have to share the supporting knowledge for unified descriptions and segmented attributions, so that the communication establishes avoiding failures to understand or misconceptions and the work-cycles progress without redundancies. The solution aims at 'intelligent' automation, based on providing information and communication services; this set-up is consistently enabled with resort to computer-integration, assuming that the individual resource is controlled by a PC and managed by a supervisor, with the basic property of granting an information frame which is taken away from human attribution and, at the same time, the meaningful process variables are transparently available by 
CAT means, with provision of monitoring maintenance, to keep optimal fit-withnorms conditions of the involved resources.

Intelligent manufacturing, thereafter, becomes powerful way for devising complex artefacts by efficient composition of high sophistication parts; some aspects, however, still hinder the spreading, such as:

- functional deficiencies of the technological equipment: presently several steps (for component sorting, for assembly combination, for approval testing, etc.) are bestowed on manual operators, particularly at critical effectiveness and versatility, when highly diversified and quickly changing product mixes are processed;

- visibility inadequacies of the issues between competing decisional steering logics: actual effects of consistent options (production programmes, on the strategic horizons; governing schedules, on the tactical horizons; management opportunities, on the execution horizons) are confined to ex post evaluation, performed by experts operating in view of empirical assessments.

Virtual testing becomes, accordingly, a broadly used opportunity for the beforehand evaluation of the 'intelligent' manufacturing options in terms, both, of the hardware set-ups actually involved and of the software outfits timely implemented.

\section{REFERENCES}

Acaccia, G.M., R.C. Michelini and R.M. Molfino (1989) Design of intelligent governors for the material-handling equipment of automated factories, in Software for Factory Automation (eds. T. Sata and G. Olling) North-Holland, Amsterdam, 297-312.

Acaccia, G.M., M. Callegari, R. Marzapani, R.C. Michelini and R.M. Molfino (1996) Development of modular assembly facilities with store-up and by-pass management, in Proc. 8th European Simulation Symposium, Genova, Oct. 2426, 289-293.

Michelini, R.C., G.M. Acaccia, M. Callegari and R.M. Molfino (1994) Flexible manufacturing with integrated control and management, in Modern Manufacturing: Control \& Technology (eds. M.B. Zaremba and B. Prasad) Springer-Verlag, London, 225-253.

Michelini, R.C., G.M. Acaccia, M. Callegari, R.M. Molfino and R.P. Razzoli (1996) Integrated product-and-process govern of robotic assembly cells, in Proc. 27th Intl. Symp. on Industrial Robots, Milano, Oct. 6-8, 415-420.

Michelini, R.C., G.M. Acaccia, M. Callegari, R.M. Molfino and R.P. Razzoli (1997) Shop controllers-managers for intelligent manufacturing, in Management and Control of Manufacturing Systems (ed. S. Tzafestas) Springer-Verlag, London, 219-254. 
dynamical system using Backlund transformations, Intl. J. Clothing Science \& Technology, vol. 8, 3, 22-42.

Rosser, P.S., Sommerfeld, J.T., Tincher, W.C. (1991) Discrete-event simulation of trouser manufacturing, Intl. J. Clothing Science \& Technology, vol. 3, 2, 1831.

Stylios, G., Sotomi, O.J., Zhu, R., Deacon, R. (1994) Intelligent garment manufacture, 75th World Conf. of the Textile Institute: 'Globalisation: Technological, Economical and Environmental Imperatives', 285-294.

Tabucanon, M.T., Estraza, M.D.E. (1989) Multi-objective production planning model for a garment factory, Intl. J. Clothing Science \& Technology, vol. 1, 3, 26-31.

Tyler, D.J., Tennent, L.F., Lowe, T.J. (1994) Simulation as training medium for industrial supervisors, The Journ. Clothing Technology \& Management, vol. $11,2,31-44$.

Taylor, P.M., Taylor, G.E., Wilkinson, A.J., Gibson, I. (1990) Mechatronics in automated apparel manufacture, I.Mech.Eng. Rpt. c419/060.

\section{BIOGRAPHY NOTE}

All Authors are at Lab. di Progettazione meccanica applicata alla Robotica industriale, Dipartimento di Meccanica e Costruzione delle Macchine, University of Genova, Italy, with research and teaching activity in robotics, quality engineering and industrial automation. 